ANNALES

POLONICI MATHEMATICI

$82.2(2003)$

\title{
3-K-contact Wolf spaces
}

\author{
by WŁodZIMIERZ JELONEK (Kraków)
}

\begin{abstract}
The aim of this paper is to give an easy explicit description of 3-K-contact structures on $\mathrm{SO}(3)$-principal fibre bundles over Wolf quaternionic Kähler manifolds.
\end{abstract}

1. Introduction. In 1965 Wolf constructed examples of symmetric quaternionic Kähler manifolds $W(G), W(G)^{*}$ associated with every simple Lie group $G$ (except $\mathrm{SU}(2)$ ). This construction is based on the properties of the highest roots in a compact, simple Lie algebra. Every space $W(G)$ is a compact symmetric space and $W(G)^{*}$ is its non-compact dual space. It has been known since $1975[\mathrm{~K}]$ that any quaternionic Kähler manifold $\left(M, g_{0}\right)$ of positive scalar curvature admits a natural $\mathrm{SO}(3)$-principal fibre bundle $p: P \rightarrow M$ such that $(P, g)$ is a 3 -Sasakian manifold and $p$ is a Riemannian submersion. However, for a long time the analogous construction for quaternionic Kähler manifolds of negative scalar curvature was not given. Recently $\mathrm{S}$. Tanno $[\mathrm{T}]$ proved that also in the case of negative scalar curvature the natural $\mathrm{SO}(3)$-principal bundle admits a structure similar to a 3-Sasakian structure, called by him the nS-structure (compare also [J-1]).

In this paper we give an elementary description of the positive and negative 3-K-contact structures related to Wolf quaternionic Kähler spaces. We show that 3 -K-contact structures are related to the real form $\mathfrak{s o}(3)_{\alpha}$ of the Lie algebra $\mathfrak{s t}(2, \mathbb{C})_{\alpha} \subset \mathfrak{g}^{\mathbb{C}}$ generated by the highest root $\alpha$ of $\mathfrak{g}$. We also give an alternative proof of the result of Bielawski [Bi] who, using Kronheimer's ideas, first explicitly described the metric of 3-Sasakian Wolf spaces (see [B$\mathrm{G}]$ ). We also remove a (slight) incorrectness of Bielawski's result (Bielawski gave the metric which is only homothetic to a 3-Sasakian metric) and give the description of negative 3-K-contact Wolf structures not considered by Bielawski. Our method is more elementary and in the spirit of Wolf's paper.

2000 Mathematics Subject Classification: 53C05, 53C20, 53C25.

Key words and phrases: quaternionic Kähler manifold, Sasakian manifold, contact manifold.

The paper was supported in part by KBN grant 2P03A 02324 . 
2. Preliminaries. For the general facts concerning 3 -K-contact and 3-Sasakian structures and quaternionic Kähler geometry we refer to [S], [B$\mathrm{G}],[\mathrm{K}],[\mathrm{Ku}],[\mathrm{Sw}],[\mathrm{T}],[\mathrm{J}-1],[\mathrm{J}-2],[\mathrm{B}]$. We shall recall several facts proved by Wolf in $[\mathrm{W}]$. Let $\mathfrak{g}$ be a compact, simple real Lie algebra and $\mathfrak{g}^{\mathbb{C}}$ its complexification. By $\langle\cdot, \cdot\rangle_{\mathrm{K}}$ we denote the Killing form on $\mathfrak{g}^{\mathbb{C}}$ and let $\sigma$ be a real structure giving a compact real form $\mathfrak{g}$ of $\mathfrak{g}^{\mathbb{C}}$. Let $\mathfrak{h}$ be a Cartan subalgebra of $\mathfrak{g}^{\mathbb{C}}$. Fix a system of roots $\Delta$ with positive roots $\Delta_{+}$. We write $\mathfrak{g}_{\beta}$ for the root space of $\beta \in \Delta$, i.e. $\mathfrak{g}_{\beta}=\left\{E \in \mathfrak{g}^{\mathbb{C}}:[H, E]=\beta(H) E\right.$ for all $\left.H \in \mathfrak{h}\right\}$. Let $\alpha \in \Delta_{+}$be a highest root; it is characterized by the condition $\left[E_{\alpha}, E_{\beta}\right]=0$ for all $\beta \in \Delta_{+}$. The following characterization of a highest root was given by Wolf $[\mathrm{W}]$ :

Proposition 1. Let $\alpha$ be a root of a complex simple Lie algebra $\mathfrak{g}^{\mathbb{C}}$ relative to a Cartan subalgebra $\mathfrak{h}$. Then $\alpha$ is the maximal root for some choice of $\Delta_{+}$if and only if the eigenvalues of $\operatorname{ad}\left(H_{\alpha}\right)$ are $-\frac{1}{2}|\alpha|^{2}, 0, \frac{1}{2}|\alpha|^{2}$ off $\mathfrak{g}_{\alpha} \oplus \mathfrak{g}_{-\alpha}$. In that case the centralizer of $H_{\alpha}$ in $\mathfrak{g}^{\mathbb{C}}$ is a direct sum $\mathfrak{z}_{1} \oplus\left\{H_{\alpha}\right\}$ of ideals, where $\mathfrak{z}_{1}$ centralizes $\mathfrak{g}_{\alpha} \oplus \mathfrak{g}_{-\alpha}$.

The centralizer $\mathfrak{z}$ of $H_{\alpha}$ in $\mathfrak{g}^{\mathbb{C}}$ is

$$
\mathfrak{z}=\mathfrak{h} \oplus \sum_{\beta \in \Phi} \mathfrak{g}_{\beta}
$$

where $\Phi=\{\beta \in \Delta:\langle\alpha, \beta\rangle=0\}$.

A quaternionic Kähler structure on a $4 n$-dimensional manifold $M, n>1$, consists of a metric $g$ and a real rank-three subbundle $\mathcal{G}$ of $\operatorname{End}(T M)$ preserved by the Levi-Civita connection and locally generated by almost Hermitian structures $I, J, K$ behaving under composition like the multiplicative pure imaginary quaternions. An equivalent definition of a quaternionic Kähler manifold $(M, g)$ is that the holonomy group of $g$ reduces to the group $\operatorname{Sp}(n) \operatorname{Sp}(1)$.

Of course, a hyper-Kähler manifold may be regarded as a special type of a quaternionic Kähler manifold with zero scalar curvature. We shall exclude this case, assuming that a quaternionic Kähler manifold is of non-zero scalar curvature.

If $n=1$ then a 4-dimensional manifold $(M, g)$ will be called quaternionic Kähler if $(M, g)$ is Einstein and self-dual with non-zero scalar curvature.

Let $(M, g)$ be a Riemannian manifold and let $\xi$ be a unit Killing vector field on $M$. Define a tensor field $\phi$ by $\phi(X)=-\nabla_{X} \xi$ and a 1 -form $\eta(X):=$ $g(\xi, X)$. Then we call $(M, g, \xi, \phi, \eta)$ a $K$-contact structure if the following relation is satisfied:

$$
\phi^{2}=-\mathrm{id}+\eta \otimes \xi .
$$

Assume that $\xi$ is a Killing vector field of unit length on $M$. We shall find conditions under which the Killing vector field $\xi$ defines a K-contact metric 
structure. Denote by $H=\operatorname{ker} \eta=\{X: g(\xi, X)=0\}$ the distribution of horizontal vectors on $M$. The following lemma is well known.

LEMma 1. Under the above assumptions the Killing vector field $\xi$ gives a K-contact structure on $M$ if and only if the tensor $J=\phi_{\mid H}$ is an almost complex structure on the bundle $H$, i.e. $J^{2}=-\mathrm{id}_{\mid H}$.

A K-contact structure $(M, g, \xi)$ is called Sasakian if

$$
R(X, \xi) Y=g(\xi, Y) X-g(X, Y) \xi
$$

where $R$ is the curvature tensor of $(M, g)$.

A Riemannian manifold $(M, g)$ with an almost complex structure $J \in$ $\operatorname{End}(T M)$ is said to be an almost Hermitian manifold if $g(J X, J Y)=$ $g(X, Y)$ for all $X, Y \in T M$. The 2-form $\Omega(X, Y)=g(J X, Y)$ is called the Kähler form of an almost Hermitian manifold $(M, g, J)$. An almost Hermitian manifold is called almost Kähler if its Kähler form is closed: $d \Omega=0$.

If $(M, g, \xi)$ is a regular $\mathrm{K}$-contact structure (i.e. there exists a quotient manifold $\left.M_{*}=M / \xi\right)$ then $\left(M, g_{*}, J_{*}\right)$ is an almost Kähler manifold, where $g_{*}$ means an induced metric and $J_{*}$ an induced almost complex structure. In that case $(M, g, \xi)$ is Sasakian if and only if $\left(M, g_{*}, J_{*}\right)$ is Kähler, i.e. if $\nabla^{*} \Omega_{*}=0$ where $\nabla^{*}$ is the Levi-Civita connection of $\left(M, g_{*}\right)$ and $\Omega_{*}$ is the Kähler form of $\left(M, g_{*}, J_{*}\right)$.

Now let us recall the definition of (positive and negative) 3-K-contact structures (see [J-1]).

Definition. Let $(P, g)$ be a Riemannian manifold that admits three distinct K-contact structures $\left(\phi_{i}, \xi_{i}, \eta_{i}\right)$ such that

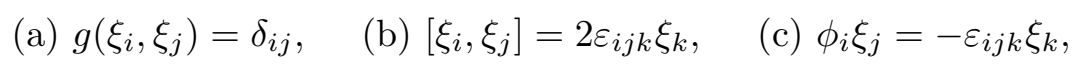

where $\phi_{i}=\nabla \xi_{i}$ and $\eta_{i}(X)=g\left(\xi_{i}, X\right)$. Denote by $H$ the horizontal distribution $H=\operatorname{ker} \eta_{1} \cap \operatorname{ker} \eta_{2} \cap \operatorname{ker} \eta_{3}=\bigcap \operatorname{ker} \eta_{i}$ and define the almost complex structures $J_{i}$ on $H$ by the formulas $J_{i}=-\phi_{i \mid H}$. We call $\left(P, \xi_{1}, \xi_{2}, \xi_{3}\right)$ a 3 -K-contact structure (or positive 3 -K-contact structure) if (for $i \neq j$ )

$$
J_{i} \circ J_{j}=\varepsilon_{i j k} J_{k}
$$

and a negative 3 -K-contact structure if (for $i \neq j$ )

$$
J_{i} \circ J_{j}=-\varepsilon_{i j k} J_{k} .
$$

A Riemannian manifold $(P, g)$ with a positive (resp. negative) 3-K-contact structure is called a positive (resp. negative) 3 -K-contact manifold. Note that arbitrary unit Killing vector fields $\xi_{i}$ satisfying $(2.1)(\mathrm{c})$ and one of conditions (2.2) define K-contact structures on $(P, g)$ (this follows from Lemma 1) so it is not necessary to include this condition in the definition above. 
If each structure $\left(M, g, \xi_{i}\right)$ is Sasakian and conditions (2.1) are satisfied then (2.2a) are automatically satisfied and we call such a positive 3 -Kcontact structure a 3-Sasakian structure.

In our paper [J-1] we have shown that if $\operatorname{dim} P \neq 11$ then every positive 3 -K-contact structure on $P$ is 3-Sasakian and every negative structure is a Tanno nS-structure.

3. 3-K-contact Wolf spaces. Let $G$ be a compact centreless Lie group. Choose a maximal torus $T$ of $G$ and denote by $\mathfrak{g}$ and $\mathfrak{t}$ the Lie algebras of $G$ and $T$ respectively. Let $\mathfrak{g}^{\mathbb{C}}$ and $\mathfrak{h}$ be the respective complexifications of $\mathfrak{g}$ and $\mathfrak{t}$. It is clear that $\mathfrak{h}$ is a Cartan subalgebra of a simple complex Lie algebra $\mathfrak{g}^{\mathbb{C}}$. Let $\Delta$ be the set of roots of $\mathfrak{g}^{\mathbb{C}}$ with respect to $\mathfrak{h}$ and fix a set of positive roots $\Delta_{+}$. Let $\alpha \in \Delta_{+}$be a highest root. Then $\alpha(X)=\left\langle H_{\alpha}, X\right\rangle$ for some $H_{\alpha} \in i \mathfrak{t} \subset \mathfrak{h}$. After rescaling the Killing form we can assume that $\langle\cdot, \cdot\rangle=$ $\left(4 /|\alpha|_{\mathrm{K}}^{2}\right)\langle\cdot, \cdot\rangle_{\mathrm{K}}$ is an ad-invariant metric on $\mathfrak{g}^{\mathbb{C}}$ such that $|\alpha|^{2}=\left\langle H_{\alpha}, H_{\alpha}\right\rangle=4$. Note that $|\alpha|_{\mathrm{K}}^{2}=\left\langle H_{\alpha}, H_{\alpha}\right\rangle>0$ and $\langle\cdot, \cdot\rangle=c\langle\cdot, \cdot\rangle_{\mathrm{K}}$ where $c>0$. We can choose vectors $E_{\alpha} \in \mathfrak{g}_{\alpha}, E_{-\alpha} \in \mathfrak{g}_{-\alpha}$ such that $E_{\alpha}=\sigma\left(E_{-\alpha}\right)$ and $\left\langle E_{\alpha}, E_{-\alpha}\right\rangle=-1$. Note that $\left\langle E_{\alpha}, E_{\alpha}\right\rangle=\left\langle E_{-\alpha}, E_{-\alpha}\right\rangle=0$. It is easy to check that $\left[E_{\alpha}, E_{-\alpha}\right]=$ $-H_{\alpha}$. Let us write $X_{\alpha}=(1 /(\sqrt{2} i))\left(E_{\alpha}-E_{-\alpha}\right), Y_{\alpha}=(1 / \sqrt{2})\left(E_{\alpha}+E_{-\alpha}\right)$, $Z_{\alpha}=\frac{1}{2} i H_{\alpha}$. Then $X_{\alpha}, Y_{\alpha}, Z_{\alpha} \in \mathfrak{g}$ and the following equalities hold:

$$
\left[X_{\alpha}, Y_{\alpha}\right]=2 Z_{\alpha}, \quad\left[X_{\alpha}, Z_{\alpha}\right]=-2 Y_{\alpha}, \quad\left[Y_{\alpha}, Z_{\alpha}\right]=2 X_{\alpha} .
$$

It follows that $\mathfrak{a}=\operatorname{span}_{\mathbb{R}}\left\{X_{\alpha}, Y_{\alpha}, Z_{\alpha}\right\}$ is a real subalgebra of $\mathfrak{g}$ isomorphic to $\mathfrak{s o}(3)$. If $\alpha$ is a highest root then $\operatorname{ad}\left(E_{\alpha}\right)^{2}=0$ and $\operatorname{ad}\left(E_{-\alpha}\right)^{2}=0$ on the space $\sum_{\beta \in \Delta_{+}, \beta \neq \alpha}\left(\mathfrak{g}_{\beta} \oplus \mathfrak{g}_{-\beta}\right)$ (see e.g. $\left.[\mathrm{K}-\mathrm{S}]\right)$.

Proposition 2. Let $\mathfrak{m}=\sum_{\beta \in \Delta_{+}, \beta \neq \alpha,\langle\beta, \alpha\rangle \neq 0} \mathfrak{g} \cap\left(\mathfrak{g}_{\beta} \oplus \mathfrak{g}_{-\beta}\right)$. Then $\operatorname{ad}\left(X_{\alpha}\right) \mathfrak{m} \subset \mathfrak{m}, \operatorname{ad}\left(Y_{\alpha}\right) \mathfrak{m} \subset \mathfrak{m}, \operatorname{ad}\left(Z_{\alpha}\right) \mathfrak{m} \subset \mathfrak{m}$ and $J_{1}=\operatorname{ad}\left(X_{\alpha}\right)_{\mid \mathfrak{m}}, J_{2}=$ $\operatorname{ad}\left(Y_{\alpha}\right)_{\mid \mathfrak{m}}, J_{3}=\operatorname{ad}\left(Z_{\alpha}\right)_{\mid \mathfrak{m}}$ define on $\mathfrak{m}$ three complex structures which give a quaternion structure on $\mathfrak{m}$, i.e. $J_{i} \circ J_{j}=\varepsilon_{i j k} J_{k}$ if $i \neq j$.

Proof. Since $X_{\alpha}, Y_{\alpha}, Z_{\alpha} \in \mathfrak{g}$ it is enough to prove that the analogous statement holds on $\mathfrak{m}^{\mathbb{C}}=\sum_{\beta \in \Delta_{+}, \beta \neq \alpha,\langle\beta, \alpha\rangle \neq 0}\left(\mathfrak{g}_{\beta} \oplus \mathfrak{g}_{-\beta}\right)$. Let $\gamma \in \Delta, \gamma \neq \alpha$ and $\langle\gamma, \alpha\rangle \neq 0$. Then $Z=\left[E_{\alpha}, E_{\gamma}\right] \in \gamma_{\alpha+\gamma}$ and if $Z \neq 0$ then $-\gamma \in \Delta_{+}$. Since from Proposition 1 we get

$$
\langle\alpha+\gamma, \alpha\rangle=\langle\alpha, \alpha\rangle+\langle\gamma, \alpha\rangle=|\alpha|^{2}-\frac{1}{2}|\alpha|^{2}=\frac{1}{2}|\alpha|^{2} \neq 0
$$

it follows that $\operatorname{ad}\left(E_{\alpha}\right) \mathfrak{m}^{\mathbb{C}} \subset \mathfrak{m}^{\mathbb{C}}$. Similarly one can prove that $\operatorname{ad}\left(E_{-\alpha}\right) \mathfrak{m}^{\mathbb{C}}$ $\subset \mathfrak{m}^{\mathbb{C}}$. Thus $\operatorname{ad}\left(X_{\alpha}\right) \mathfrak{m}^{\mathbb{C}} \subset \mathfrak{m}^{\mathbb{C}}$ and $\operatorname{ad}\left(Y_{\alpha}\right) \mathfrak{m}^{\mathbb{C}} \subset \mathfrak{m}^{\mathbb{C}}$. Note that $\operatorname{ad}\left(H_{\alpha}\right) E_{\gamma}=$ $\langle\alpha, \gamma\rangle E_{\gamma}= \pm \frac{1}{2}|\alpha|^{2} E_{\gamma}$. Thus $\operatorname{ad}\left(Z_{\alpha}\right) E_{\gamma}= \pm i E_{\gamma}$ and $J_{3}^{2}=-\mathrm{id}_{\mathfrak{m}}$. We also have

$\operatorname{ad}\left(E_{\alpha}+E_{-\alpha}\right)^{2}=\operatorname{ad}\left(E_{\alpha}\right)^{2}+\operatorname{ad}\left(E_{-\alpha}\right) \circ \operatorname{ad}\left(E_{\alpha}\right)+\operatorname{ad}\left(E_{\alpha}\right) \circ \operatorname{ad}\left(E_{-\alpha}\right)+\operatorname{ad}\left(E_{-\alpha}\right)^{2}$. 
Both $\operatorname{ad}\left(E_{\alpha}\right)^{2}$ and $\operatorname{ad}\left(E_{-\alpha}\right)^{2}$ vanish on $\mathfrak{m}^{\mathbb{C}}$. Hence $\operatorname{ad}\left(E_{\alpha}+E_{-\alpha}\right)^{2}\left(E_{\gamma}\right)=$ $\left[E_{\alpha},\left[E_{-\alpha}, E_{\gamma}\right]\right]+\left[E_{-\alpha},\left[E_{\alpha}, E_{\gamma}\right]\right]$.

Now assume that $\gamma \in \Delta_{+}$. Then

$$
\begin{aligned}
{\left[E_{\alpha},\left[E_{-\alpha}, E_{\gamma}\right]\right]+\left[E_{-\alpha},\left[E_{\alpha}, E_{\gamma}\right]\right] } & =-\left[E_{\gamma},\left[E_{\alpha}, E_{-\alpha}\right]\right]+2\left[E_{-\alpha},\left[E_{\alpha}, E_{\gamma}\right]\right] \\
& =-\left[E_{\gamma},\left[E_{\alpha}, E_{-\alpha}\right]\right]=\left[E_{\gamma}, H_{\alpha}\right] \\
& =-\langle\alpha, \gamma\rangle E_{\gamma}=-\frac{1}{2}|\alpha|^{2} E_{\gamma}
\end{aligned}
$$

where we used the fact that if $\alpha \in \Delta_{+}$is a highest root and $\gamma \in \Delta_{+}$, $\langle\alpha, \gamma\rangle \neq 0$ then $\langle\alpha, \gamma\rangle>0$ since otherwise $\alpha+\gamma$ would be a positive root, a contradiction. If $-\gamma \in \Delta_{+}$then

$$
\begin{aligned}
{\left[E_{\alpha},\left[E_{-\alpha}, E_{\gamma}\right]\right]+\left[E_{-\alpha},\left[E_{\alpha}, E_{\gamma}\right]\right] } & =-\left[E_{\gamma},\left[E_{-\alpha}, E_{\alpha}\right]\right]+2\left[E_{\alpha},\left[E_{\alpha}, E_{\gamma}\right]\right] \\
& =\left[E_{\gamma},\left[E_{\alpha}, E_{-\alpha}\right]\right]=-\left[E_{\gamma}, H_{\alpha}\right] \\
& =\langle\alpha, \gamma\rangle E_{\gamma}=-\frac{1}{2}|\alpha|^{2} E_{\gamma} .
\end{aligned}
$$

Recall that $|\alpha|^{2}=4$. Consequently, $\operatorname{ad}\left(E_{\alpha}+E_{-\alpha}\right)_{\mid \mathfrak{m}}^{2}=-2 \mathrm{id}_{\mid \mathfrak{m}}$ and $\operatorname{ad}\left(Y_{\alpha}\right)_{\mid \mathfrak{m}}^{2}$ $=-\mathrm{id}_{\mid \mathfrak{m}}$. Thus $J_{2}^{2}=-\mathrm{id}_{\mid \mathfrak{m}}$. Analogously one can prove that $J_{1}^{2}=-\mathrm{id}_{\mid \mathfrak{m}}$.

Now we show that $J_{1} \circ J_{2}=-J_{3}$. We have

$$
\begin{aligned}
{\left[E_{\alpha}-E_{-\alpha},\left[E_{\alpha}+E_{-\alpha}, E_{\gamma}\right]\right] } & =\left[E_{\alpha},\left[E_{-\alpha}, E_{\gamma}\right]\right]-\left[E_{-\alpha},\left[E_{\alpha}, E_{\gamma}\right]\right] \\
& =-\left[E_{\gamma},\left[E_{\alpha}, E_{-\alpha}\right]\right]=-\left[H_{\alpha}, E_{\gamma}\right]
\end{aligned}
$$

and consequently $J_{1} \circ J_{2}=J_{3}$. It follows easily that $J_{i} \circ J_{j}=\varepsilon_{i j k} J_{k}$ if $i \neq j$.

Now consider the group $G$ with the bi-invariant metric $g$ induced by $-\langle\cdot, \cdot\rangle$. Note that $g$ is positive definite. Write $\mathfrak{l}=\{H \in \mathfrak{t}: \alpha(H)=0\} \oplus$ $\sum_{\beta \in \Delta_{+},\langle\alpha, \beta\rangle=0} \mathfrak{g} \cap\left(\mathfrak{g}_{\beta} \oplus \mathfrak{g}_{-\beta}\right)$. Then $\mathfrak{l}$ is a Lie subalgebra of $\mathfrak{g}$ and $[\mathfrak{a}, \mathfrak{l}]=0$. Note that $\mathfrak{g}=\mathfrak{l} \oplus \mathfrak{a} \oplus \mathfrak{m}$ and $[\mathfrak{l} \oplus \mathfrak{a}, \mathfrak{m}] \subset \mathfrak{m},[\mathfrak{m}, \mathfrak{m}] \subset \mathfrak{l} \oplus \mathfrak{a}$. Let $L, A$ be the connected subgroups of $G$ corresponding to the Lie subalgebras $\mathfrak{l}, \mathfrak{a}$ respectively. Let $t>0$ and let $g_{t}$ be a left-invariant metric on $G$ defined by (we identify $g_{t}$ with a metric on $\left.\mathfrak{g}\right) g_{t}=g_{0}+g_{1}+t g_{2}$ where $g_{0}=g_{\mid \mathfrak{r}}$, $g_{1}=g_{\mid \mathfrak{a}}, g_{2}=g_{\mid \mathfrak{m}}$. Let $p: G \rightarrow G / L$ be the natural projection. Then the metric $g_{1}+t g_{2}$ on $\mathfrak{a} \oplus \mathfrak{m}$ induces an invariant metric $h_{t}$ on the coset space $G / L$ such that $p:\left(G, g_{t}\right) \rightarrow\left(G / L, h_{t}\right)$ is a Riemannian submersion. The left-invariant vector fields $X_{\alpha}, Y_{\alpha}, Z_{\alpha} \in \mathfrak{g}$ are Killing vector fields with respect to the metric $g_{t}$.

In fact it is easy to check that if $A \in \mathfrak{a}$ then $g_{t}(\operatorname{ad}(A) X, Y)+g_{t}(X, \operatorname{ad}(A) Y)$ $=0$ for all $X, Y \in \mathfrak{g}$. Since they are horizontal with respect to the Riemannian submersion $p$ and $[\mathfrak{l}, \mathfrak{a}]=0$ it follows that there exist Killing vector fields $\xi_{1}, \xi_{2}, \xi_{3}$ on $G / L$ which are $p$-related to $X_{\alpha}, Y_{\alpha}, Z_{\alpha}$ respectively.

We show that for an appropriate choice of $t$ the fields $\xi_{1}, \xi_{2}, \xi_{3}$ define on $M=G / L$ a positive 3 -K-contact structure (in fact Sasakian). Define the 
three 1-forms on $G$ by

$$
\theta_{1}(X)=g_{t}\left(X_{\alpha}, X\right), \quad \theta_{2}(X)=g_{t}\left(Y_{\alpha}, X\right), \quad \theta_{3}(X)=g_{t}\left(Z_{\alpha}, X\right) .
$$

Note that the forms $\theta_{i}$ are left-invariant, $\theta_{i}(Y)=0$ if $Y \in \mathfrak{l}$ and $\operatorname{ad}_{l}^{*}\left(\theta_{i}\right)=\theta_{i}$ for any $l \in L$. Thus (see for example [O-T, p. 139]) $\theta_{i}=p^{*} \eta_{i}$ where $\eta_{i}$ are the one-forms on $M$ defined by $\eta_{i}(X)=h_{t}\left(\xi_{i}, X\right)$. Let $X, Y \in \mathfrak{l} \oplus \mathfrak{a}$. Then

$$
d \theta_{i}(X, Y)=-\theta_{i}([X, Y]) .
$$

The group $A$ is a totally geodesic subgroup of $\left(G, g_{t}\right)$. Consequently, the orbits of the action of $A$ on $M$ are totally geodesic submanifolds of $M$ (the fundamental Killing vector fields of $A$ have constant length). From (3.2) we get (setting $T_{1}=X_{\alpha}, T_{2}=Y_{\alpha}, T_{3}=Z_{\alpha}$ )

$$
d \theta_{i}(X, Y)=-g_{t}\left(T_{i},[X, Y]\right) \text {. }
$$

Note that $d \theta_{i}(X, Y)=0$ if $X \in \mathfrak{a}$ and $Y \in \mathfrak{m}$. We also have

$$
\begin{array}{r}
d \theta_{i}(X, Y)=\left\langle T_{i},[X, Y]\right\rangle=-\left\langle\operatorname{ad}(X) T_{i}, Y\right\rangle=g_{t}\left(\operatorname{ad}(X) T_{i}, Y\right) \\
\text { if } X, Y \in \mathfrak{a}, \\
d \theta_{i}(X, Y)=\left\langle T_{i},[X, Y]\right\rangle=-\left\langle\operatorname{ad}(X) T_{i}, Y\right\rangle=\frac{1}{t} g_{t}\left(\operatorname{ad}(X) T_{i}, Y\right) \\
\text { if } X, Y \in \mathfrak{m} .
\end{array}
$$

Thus if $X, Y \in \mathfrak{m} \oplus \mathfrak{a}$ and $g \in G$ and $x=p(g) \in M$ then $p\left(X_{g}\right) \in T_{x} M$, $p\left(Y_{g}\right) \in T_{x} M$ and

$$
\begin{array}{rlrl}
d \eta_{i}(p(X), p(Y))_{x)} & =g_{t}\left(\operatorname{ad}(X) T_{i}, Y\right) & & \text { if } X, Y \in \mathfrak{a}, \\
d \eta_{i}(p(X), p(Y))_{x}=\frac{1}{t} g_{t}\left(\operatorname{ad}(X) T_{i}, Y\right) & & \text { if } X, Y \in \mathfrak{m} .
\end{array}
$$

Consequently, since $p^{*} d \eta_{i}=d \theta_{i}$ and $d \eta_{i}(X, Y)=2 h_{t}\left(\nabla_{X}^{t} \xi_{i}, Y\right)$ we obtain (note that $p:\left(G, g_{t}\right) \rightarrow\left(M, h_{t}\right)$ is a Riemannian submersion)

$$
\begin{array}{ll}
\nabla_{p(X)}^{t} \xi_{i}=-\frac{1}{2} p\left(\operatorname{ad}\left(T_{i}\right)(X)\right) & \text { if } X \in \mathfrak{a}, \\
\nabla_{p(X)}^{t} \xi_{i}=-\frac{1}{2 t} p\left(\operatorname{ad}\left(T_{i}\right)(X)\right) & \text { if } X \in \mathfrak{m},
\end{array}
$$

where by $\nabla^{t}$ we denote the Levi-Civita connection of $\left(M, h_{t}\right)$. If we identify the space $T_{x} M$ with $\mathfrak{a} \oplus \mathfrak{m}$ by means of $p$ then

$$
\begin{aligned}
\nabla^{t} \xi_{i \mid \mathfrak{a}} & =-\frac{1}{2} \operatorname{ad}\left(T_{i}\right)_{\mid \mathfrak{a}}, \\
\nabla^{t} \xi_{i \mid \mathfrak{m}} & =-\frac{1}{2 t} \operatorname{ad}\left(T_{i}\right)_{\mid \mathfrak{m}} .
\end{aligned}
$$

Note that if $p(g)=p\left(g_{1}\right)$ then $g_{1}=g l$ where $l \in L$. Thus if we identify $(\mathfrak{a} \oplus \mathfrak{m})_{g}=d_{e} L_{g}(\mathfrak{a} \oplus \mathfrak{m}) \subset T_{g} G$ and $(\mathfrak{a} \oplus \mathfrak{m})_{g_{1}}=d_{e} L_{g_{1}}(\mathfrak{a} \oplus \mathfrak{m}) \subset T_{g_{1}} G$ with $T_{g L} G / L$ by means of $p$ and $X \in \mathfrak{a} \oplus \mathfrak{m}$ then a vector $p\left(X_{g}\right) \in T_{g L} G / L$ is 
represented by a vector $(\operatorname{Ad}(l) X)_{g_{1}} \in \mathfrak{m}_{g_{1}}$. However $[L, A]=\{e\}$ and consequently (3.7) does not depend on the choice of the isomorphism $(\mathfrak{a} \oplus \mathfrak{m})_{g}=$ $T_{g L} G / L$.

Now consider the Lie algebra $\mathfrak{g}_{0}=\mathfrak{l} \oplus \mathfrak{a} \oplus i \mathfrak{m} \subset \mathfrak{g}^{\mathbb{C}}$. To this Lie algebra corresponds a connected Lie subgroup $G_{0}$ of the Lie group $G^{\mathbb{C}}$. We call $G_{0}$ the dual group of $G$. On the group $G^{\mathbb{C}}$ we have a bi-invariant metric $g$ induced by the Killing form $\langle\cdot, \cdot\rangle_{\mathrm{K}}$ on $g^{\mathbb{C}}$, i.e.

$$
g(X, Y)_{e}=-\frac{1}{|\alpha|^{2}}\langle X, Y\rangle_{\mathrm{K}}=-\langle X, Y\rangle .
$$

Let $t>0$ and let $g_{t}$ be a left-invariant metric on $G_{0}$ defined by (we identify $g_{t}$ with a metric on $\left.\mathfrak{g}_{0}\right) g_{t}=g_{0}+g_{1}+t g_{2}$ where $g_{0}=g_{\mid \mathfrak{l}}, g_{1}=g_{\mid \mathfrak{a}}, g_{2}=-g_{\mid i \mathfrak{m}}$. Note that $g_{t}$ is a positive-definite metric on $G_{0}$. Let $p_{0}: G_{0} \rightarrow G_{0} / L$ be a natural projection. Then the metric $g_{1}+t g_{2}$ on $\mathfrak{a} \oplus \mathfrak{m}$ induces an invariant metric $h_{t}$ on the coset space $G_{0} / L$ such that $p_{0}:\left(G_{0}, g_{t}\right) \rightarrow\left(G_{0} / L, h_{t}\right)$ is a Riemannian submersion. The left-invariant vector fields $X_{\alpha}, Y_{\alpha}, Z_{\alpha} \in \mathfrak{a} \subset \mathfrak{g}_{0}$ are Killing vector fields with respect to the metric $g_{t}$ on $G_{0}$. It follows that there exist Killing vector fields $\xi_{1}, \xi_{2}, \xi_{3}$ on $M_{0}=G_{0} / L$ which are $p_{0}$-related to $T_{1}, T_{2}, T_{3}$ respectively.

Define three 1-forms on $G$ by $\theta_{i}(X)=g_{t}\left(T_{i}, X\right)$. Note that the forms $\theta_{i}$ are left-invariant, $\theta_{i}(Y)=0$ if $Y \in \mathfrak{l}$ and $\operatorname{ad}_{l}^{*} \theta_{i}=\theta_{i}$ for any $l \in L$. Thus $\theta_{i}=p^{*} \eta_{i}$ where $\eta_{i}$ are one-forms on $M$ defined by $\eta_{i}(X)=h_{t}\left(\xi_{i}, X\right)$. Let $X, Y \in \mathfrak{l} \oplus \mathfrak{a}$. Then as above

$$
d \theta_{i}(X, Y)=-\theta_{i}([X, Y])=-g_{t}\left(T_{i},[X, Y]\right) .
$$

Note that $d \theta_{i}(X, Y)=0$ if $X \in \mathfrak{a}$ and $Y \in i \mathfrak{m}$. We also have

$$
\begin{array}{r}
d \theta_{i}(X, Y)=\left\langle T_{i},[X, Y]\right\rangle=-\left\langle\operatorname{ad}(X) T_{i}, Y\right\rangle=g_{t}\left(\operatorname{ad}(X) T_{i}, Y\right) \\
\text { if } X, Y \in \mathfrak{a}, \\
d \theta_{i}(X, Y)=\left\langle T_{i},[X, Y]\right\rangle=-\left\langle\operatorname{ad}(X) T_{i}, Y\right\rangle=-\frac{1}{t} g_{t}\left(\operatorname{ad}(X) T_{i}, Y\right) \\
\text { if } X, Y \in i \mathfrak{m} .
\end{array}
$$

Thus if $X, Y \in i \mathfrak{m} \oplus \mathfrak{a}$ and $g \in G_{0}$ and $x=p_{0}(g) \in M_{0}$ then $p_{0}\left(X_{g}\right) \in T_{x} M_{0}$, $p_{0}\left(Y_{g}\right) \in T_{x} M_{0}$ and

$$
\begin{aligned}
d \eta_{i}\left(p_{0}(X), p_{0}(Y)\right)_{x)}=g_{t}\left(\operatorname{ad}(X) T_{i}, Y\right) & \text { if } X, Y \in \mathfrak{a}, \\
d \eta_{i}\left(p_{0}(X), p_{0}(Y)\right)_{x}=-\frac{1}{t} g_{t}\left(\operatorname{ad}(X) T_{i}, Y\right) & \text { if } X, Y \in i \mathfrak{m} .
\end{aligned}
$$

Consequently, since $d \eta_{i}=p_{0}^{*} d \theta_{i}$ and $d \eta_{i}(X, Y)=2 h_{t}\left(\nabla_{X}^{t} \xi_{i}, Y\right)$ we obtain (note that $p_{0}:\left(G_{0}, g_{t}\right) \rightarrow\left(M_{0}, h_{t}\right)$ is a Riemannian submersion and we identify $T M_{0}$ with $\mathfrak{a} \oplus i \mathfrak{m}$ by means of $\left.p_{0}\right)$

$$
\nabla^{t} \xi_{i \mid \mathfrak{a}}=-\frac{1}{2} \operatorname{ad}\left(T_{i}\right)_{\mid \mathfrak{a}},
$$




$$
\nabla^{t} \xi_{i \mid i \mathfrak{m}}=\frac{1}{2 t} \operatorname{ad}\left(T_{i}\right)_{\mid i \mathfrak{m}}
$$

Hence we can prove

TheOREM 1. Let $G$ be a compact, simple and centreless Lie group and let $G_{0}$ be its dual group. Then $\left(G / L, h_{t}, \xi_{1}, \xi_{2}, \xi_{3}\right)$ is a positive 3 -K-contact structure and $\left(G_{0} / L, h_{t}, \xi_{1}, \xi_{2}, \xi_{3}\right)$ is a negative 3 -K-contact structure if and only if $t=1 / 2$.

Proof. Note that in both cases considered above we have $\nabla_{\xi_{i}}^{t} \xi_{j}=\varepsilon_{i j k} \xi_{k}$. Thus conditions (2.1) of the definition of 3-K-contact structure are satisfied. If we identify $T_{x} M$ with $\mathfrak{a} \oplus \mathfrak{m}$ (respectively $T_{x} M_{0}$ with $\mathfrak{a} \oplus i \mathfrak{m}$ ) by means of $p$ (resp. $p_{0}$ ) then the space $H$ described in the definition coincides with $\mathfrak{m}$ (resp. $i \mathfrak{m}$ ). With this identification $J_{i}=\nabla^{t} \xi_{i \mid H}$ equals

$$
\nabla^{t} \xi_{i \mid \mathfrak{m}}=-\frac{1}{2 t} \operatorname{ad}\left(T_{i}\right)_{\mid \mathfrak{m}}
$$

in the first case and

$$
\nabla^{t} \xi_{i \mid i \mathfrak{m}}=\frac{1}{2 t} \operatorname{ad}\left(T_{i}\right)_{\mid i \mathfrak{m}}
$$

in the second case. From Proposition 2 it follows that if $t=1 / 2$ then $\nabla^{t} \xi_{i}$ defines on the space $H_{i}=\left\{X \in T M\left(T M_{0}\right): h_{t}\left(\xi_{i}, X\right)=0\right\}$ an almost complex structure (i.e. $\left.\left(\nabla^{t} \xi_{i \mid H_{i}}\right)^{2}=-\mathrm{id}_{H_{i}}\right)$. Consequently, each field $\xi_{i}$ defines a K-contact structure on $\left(M, h_{1 / 2}\right)$ (resp. on $\left.\left(M_{0}, h_{1 / 2}\right)\right)$.

Now from (3.7) and (3.11) it follows that for $t=1 / 2$ we have

$$
-\nabla^{t} \xi_{i \mid \mathfrak{m}}=\frac{1}{2 t} \operatorname{ad}\left(T_{i}\right)_{\mid \mathfrak{m}}=J_{i}
$$

and respectively

$$
-\nabla^{t} \xi_{i \mid i \mathfrak{m}}=-\frac{1}{2 t} \operatorname{ad}\left(T_{i}\right)_{\mid i \mathfrak{m}}=i J_{i} i
$$

where $J_{i}$ is defined in Proposition 2 and $i J_{i} i(X)=i\left(J_{i}(i X)\right)$ for $X \in i \mathfrak{m}$. Consequently, it follows from Proposition 2 that $\left(M, h_{1 / 2}, \xi_{1}, \xi_{2}, \xi_{3}\right)$ is a positive 3 -K-contact structure and that $\left(M_{0}, h_{1 / 2}, \xi_{1}, \xi_{2}, \xi_{3}\right)$ is a negative 3 -K-contact structure.

Note that the spaces $G / L$ are $\mathrm{SO}(3)$ or $\mathrm{Sp}(1)$ bundles over the symmetric quaternionic spaces $W(G)$, and $G / L$ are exactly the spaces

$$
\begin{gathered}
\mathrm{Sp}(n) / \mathrm{Sp}(n-1)=S^{4 n-1}, \quad \mathrm{SU}(m) / S(U(m-2) \times U(1)), \\
\mathrm{SO}(k) / \mathrm{SO}(k-4) \times \mathrm{Sp}(1), \quad G_{2} / \mathrm{Sp}(1), \quad F_{4} / \mathrm{Sp}(3), \\
E_{6} / \mathrm{SU}(6), \quad E_{7} / \operatorname{Spin}(12), \quad E_{8} / E_{7},
\end{gathered}
$$

where $n \geq 1, m \geq 3, k \geq 7$, and $G / L$ is an $\operatorname{Sp}(1)$ bundle only in the first case of $\operatorname{Sp}(n) / \operatorname{Sp}(n-1)=S^{4 n-1}$. Note that this space admits a $\mathbb{Z}_{2}$ quotient $\operatorname{Sp}(n) / \operatorname{Sp}(n-1) \times \mathbb{Z}_{2}=\mathbb{R} \mathbb{P}^{4 n-1}$ which is also a 3 -Sasakian space. 
The holonomy representation of $W(G)$ with symmetric metric is the representation ad of the group $L A$ on the space $\mathfrak{m}$ with quaternionic structure given by $J_{1}, J_{2}, J_{3}$ where $A=\operatorname{Sp}(1)$ and the action $A \ni a \mapsto \operatorname{Ad}(a)_{\mid \mathfrak{m}}$ coincides with the standard representation of the group $\operatorname{Sp}(1)=\{q \in \mathbb{H}: q \bar{q}=1\}$ on the space $\mathbb{H}^{n}$ where $n=\frac{1}{4} \operatorname{dim} \mathfrak{m}$. Consequently, $L \operatorname{Sp}(1) \subset \operatorname{Sp}(n) \operatorname{Sp}(1)$.

Now our aim is to give a precise description of twistor spaces of Wolf spaces (see $[\mathrm{S}],[\mathrm{Sw}],[\mathrm{J}-1]$ ). In the negative case we obtain homogeneous almost Kähler manifolds which are not Kähler. In the positive case we get Einstein Kähler spaces $G / L T$ of positive scalar curvature where $T$ is the one-dimensional torus group. We only give the proof for the negative case, the positive case being similar.

Proposition 3. The homogeneous spaces $G_{0} / L T$, where $T$ is the oneparameter subgroup of $\mathrm{Sp}(1)=A$ generated by $Z_{\alpha} \in \mathfrak{a}$ with metric induced by the metric $m=g_{\mid \mathfrak{a}_{1}}-\frac{1}{2} g_{\mid i \mathfrak{m}}$ on the space $\mathfrak{m}_{0}=\mathfrak{a}_{1} \oplus$ im where $g=-\left(4 /|\alpha|_{\mathrm{K}}^{2}\right)\langle\cdot, \cdot\rangle_{\mathrm{K}}$ and $\mathfrak{a}_{1}=\operatorname{span}_{\mathbb{R}}\left\{X_{\alpha}, Y_{\alpha}\right\}$, are strictly almost Kähler homogeneous spaces.

Proof. Let $\pi_{*}$ be the natural projection $\pi_{*}: G_{0} / L \rightarrow G_{0} / L T$. Since $G_{0} / L T$ is the quotient of $G_{0} / L$ by the one-parameter group of isometries generated by the Killing vector field $\xi_{3}$ and $\left(\left(G_{0} / L, h_{1 / 2}\right), \xi_{3}\right)$ is a K-contact structure it follows that $G_{0} / L T$ with the induced metric $g_{*}$ and an almost Hermitian structure $J_{*}$ such that $g\left(J_{*} \pi_{*} X, \pi_{*}(Y)\right)=d \eta_{1}(X, Y)$ is an almost Kähler manifold with a Kähler form $\Omega_{*}(X, Y)=g_{*}\left(J_{*} X, Y\right)$. It is not Sasakian, since $R\left(X, \xi_{i}\right) \xi_{j}=2 \varepsilon_{i j k} \phi_{k}(X)$ for a horizontal vector $X$.

REMARK. Note that the metric on the 3 -K-contact space is uniquely determined as $g_{t}=g_{0}+g_{1}+t g_{2}$ where $g=\left(4 /|\alpha|_{\mathrm{K}}^{2}\right)\langle\cdot, \cdot\rangle_{\mathrm{K}}, g_{0}=g_{\mid \mathfrak{l}}, g_{1}=g_{\mid \mathfrak{a}}$, $g_{2}=\varepsilon g_{\mid \mathfrak{m}_{1}}$ with $\varepsilon=1$ and $\mathfrak{m}_{1}=\mathfrak{m}$ in the case of a positive 3 -K-contact space $G / L$ and $\varepsilon=-1$ and $\mathfrak{m}_{1}=i \mathfrak{m}$ in the case of a negative 3 -K-contact space $G_{0} / L$, whereas the metric on the almost Kähler space $G_{0} / L T$ is given up to homothety, i.e. we can also choose the metric $m=g_{\mid \mathfrak{a}_{1}}-\frac{1}{2} g_{\mid i \mathfrak{m}}$ on the space $\mathfrak{m}_{0}=\mathfrak{a}_{1} \oplus i \mathfrak{m}$ where $g=-\langle\cdot, \cdot\rangle_{\mathrm{K}}$ and the twistor space with this metric is still almost Kähler.

Our last aim is to give a precise description of the reduction of the principal bundle $\mathrm{SO}(M)$ of orthonormal oriented frames of the Wolf spaces $W(G), W(G)^{*}$ to the $L A$-structure $P(L A, M)$, and to describe the LeviCivita connection in $P$. We denote by $g_{*}$ the symmetric metric on $W(G)$ or $W(G)^{*}$, i.e. $g_{*}$ is induced by the metric $-\langle\cdot, \cdot\rangle$ on $\mathfrak{m}$ or $\langle\cdot, \cdot\rangle$ on $i \mathfrak{m}$. Write $K=L A$ and denote by $\mathfrak{k}$ the Lie algebra of $K$. By $\pi: G / K \rightarrow M$ or $\pi: G_{0} / K \rightarrow M$ we mean the natural projection.

Define $P=G$ in the positive case and $P=G_{0}$ in the negative case. Define the horizontal distribution $\mathcal{H} \subset T G$ (resp. $\left.T G_{0}\right)$ by $\mathcal{H}_{g}=d_{e} L_{g}\left(\mathfrak{m}_{1}\right)$ and the 
vertical distribution by $\mathcal{V}_{g}=d_{e} L_{g}(\mathfrak{k})$. Let $\theta^{\mathrm{C}}$ be a Cartan form on $G$ (resp. $\left.G_{0}\right)$ defined on $X \in T_{g} G$ (resp. $\left.T_{g} G_{0}\right)$ as follows: $\theta^{\mathrm{C}}(X)=d_{e} L_{g^{-1}}(X) \in \mathfrak{g}$ $\left(\right.$ resp $\left.\mathfrak{g}_{0}\right)$. Denote by $p_{\mathfrak{k}}, p_{\mathfrak{m}_{1}}$ the projections onto $\mathfrak{k}, \mathfrak{m}_{1}$ with respect to the decomposition $\mathfrak{g}=\mathfrak{k} \oplus \mathfrak{m}$ and $\mathfrak{g}_{0}=\mathfrak{k} \oplus i \mathfrak{m}$. Then the connection form $\omega$ with horizontal distribution $\mathcal{H}$ is defined by $\omega=p_{\mathfrak{k}} \circ \theta^{\mathrm{C}}$.

We shall treat $G$ (resp. $G_{0}$ ) as a subbundle of the bundle $\mathrm{SO}(M)$ by identifying an element $g \in G$ with the mapping $u_{g}: \mathfrak{m}_{1} \rightarrow T_{g K} M$ given by $u_{g}(X)=\pi\left(d_{e} L_{g}(X)\right)$. Then the canonical form of $P \subset \mathrm{SO}(M)$ is $\theta=$ $p_{\mathfrak{m}_{1}} \circ \theta^{\mathrm{C}}(X)$ since

$$
\theta(X)=u_{g}^{-1}(\pi(X))=p_{\mathfrak{m}_{1}} \circ \theta^{\mathrm{C}}(X) .
$$

Since $\left[\mathfrak{m}_{1}, \mathfrak{m}_{1}\right] \subset \mathfrak{k}$ it follows easily that $\Theta(X, Y)=d \theta(h X, h Y)=0$ where $h X \in \mathcal{H}$ denotes the $\mathcal{H}$-component of $X$ with respect to the decomposition $T G=\mathcal{V} \oplus \mathcal{H}$.

Thus the connection $\Gamma$ given by $\omega$ is a torsionless connection in the principal bundle of oriented orthonormal frames, i.e. $\Gamma$ is the Levi-Civita connection of $\left(M, g_{*}\right)$. Note that we treat $K$ as a subgroup of $\mathrm{SO}\left(\mathfrak{m}_{1}\right)$ (where on $\mathfrak{m}_{1}$ we have the metric $-\langle\cdot, \cdot\rangle$ if $\mathfrak{m}_{1}=\mathfrak{m}$, and $\langle\cdot, \cdot\rangle$ if $\left.\mathfrak{m}_{1}=i \mathfrak{m}\right)$, via the representation $\mathrm{Ad}: K \rightarrow \mathrm{SO}\left(\mathfrak{m}_{1}\right)$.

Let $G_{1}=G$ or $G_{1}=G_{0}$ and $(a, b) \ni t \mapsto x_{t} \in M$ be a smooth path in $M$ such that

$$
x(a)=e K=x_{0} \in G_{1} / K=M
$$

and let $Y \in T_{x_{0}} M$. Then there exists $Y^{*} \in \mathfrak{m}_{1} \subset \mathfrak{g}=T_{e} G_{1}$ such that $Y=\pi\left(Y^{*}\right)$. Let $g_{t}$ be a horizontal lift of $x_{t}$ to the $K$-principal bundle $G_{1}$ over $M$ with connection $\Gamma$, i.e. $\pi\left(g_{t}\right)=x_{t}$ and $\omega\left(\dot{g}_{t}\right)=0$. Then $Y_{t}=\pi\left(Y_{t}^{*}\right)$ is a parallel field along $x_{t}$ where $Y_{t}^{*}=d_{e} L_{g_{t}}\left(Y^{*}\right) \in \mathcal{H}_{g_{t}}$. Note that $Y_{a}=Y$. If $x_{a}=x_{b}=x_{0}$ then $g_{b}=k \in K$ and under the identification $\mathcal{H}_{e}=\mathcal{H}_{k}$ we obtain $Y_{b}^{*}=\operatorname{ad}(k) Y^{*}$.

Consequently, the holonomy group coincides exactly with $K$ and the holonomy representation is $K \ni k \mapsto \operatorname{ad}(k)_{\mid \mathfrak{m}_{1}} \in \mathrm{SO}\left(\mathfrak{m}_{1}\right)$ (for the details see [H, p. 207]). Recall that the endomorphisms $J_{i}: \mathfrak{m}_{1} \rightarrow \mathfrak{m}_{1}$ are described in Proposition 2. It is easy to see that the bundle $\mathcal{G} \subset \operatorname{End}(T M)$ of endomorphisms defining the quaternionic structure on $M$ is generated by the endomorphisms $\pi\left(J_{i} \circ u_{g}^{-1}\right)$ where $u_{g} \in G_{1}, i \in\{1,2,3\}$ (see the construction of $\mathcal{G}$ in $[\mathrm{J}-1],[\mathrm{J}-2])$. We have

Proposition 4. The principal bundle $\mathrm{SO}(M)$ and the Levi-Civita connection of a quaternionic Kähler Wolf space $W(G)\left(\right.$ resp. $\left.W(G)^{*}\right)$ admit a reduction to a $K$-structure $G_{1} \subset \mathrm{SO}(M)$ with Levi-Civita connection form $\omega=p_{\mathfrak{k}} \circ \theta^{\mathrm{C}}$. The bundle $\mathcal{G}$ is generated by the endomorphisms $\pi\left(J_{i} \circ u_{g}^{-1}\right)$ where $i \in\{1,2,3\}$. 
It follows that our construction of positive and negative 3-K-contact structures coincides with the one given in [J-2]. The only difference is that we consider a $K$-reduction $G, G_{0} \subset \mathrm{SO}(M)$ instead of an $\operatorname{Sp}(n) \operatorname{Sp}(1)$-reduction $Q \subset \mathrm{SO}(M)$. It is clear that $K \subset \mathrm{Sp}(n) \mathrm{Sp}(1)$. Consequently, the positive structure is 3-Sasakian and the negative structure is the Tanno nS-structure. In the case $4 n \neq 8$ this also follows directly from [J-1]. Let us remark here that $K$ coincides with $\operatorname{Sp}(n) \operatorname{Sp}(1)$ only in the case of $M=\mathbb{H} \mathbb{P}^{n}=$ $\operatorname{Sp}(n+1) / \operatorname{Sp}(n) \operatorname{Sp}(1)$ and its dual Wolf space (see $[\mathrm{A}])$.

\section{References}

[A] D. V. Alekseevskiı̌, Compact quaternion spaces, Funktsional. Anal. i Prilozhen. 2 (1968), no. 2, 11-20 (in Russian).

[B] A. Besse, Einstein Manifolds, Springer, Berlin, 1987.

[Bi] R. Bielawski, On the hyperkähler metrics associated to singularities of nilpotent varieties, Ann. Global Anal. Geom. 14 (1996), 177-191

[B-G] P. Boyer and K. Galicki, 3-Sasakian manifolds, in: Surveys in Differential Geometry: Essays on Einstein Manifolds, Surveys Differential Geom. 6, Internat. Press, Boston, 1999, 123-184.

[E-S] J. Eells et S. Salamon, Constructions twistorielles des applications harmoniques, C. R. Acad. Sci. Paris Sér. I 296 (1983), 685-687.

[G-L] K. Galicki and H. B. Lawson, Quaternionic reduction and quaternionic orbifolds, Math. Ann. 282 (1988), 1-21.

[H] S. Helgason, Differential Geometry and Symmetric Spaces, Academic Press, New York, 1962.

[J-1] W. Jelonek, Positive and negative 3-K-contact structures, Proc. Amer. Math. Soc. 120 (2001), 247-256.

[J-2] -, Quaternionic-Kähler geometry and almost Kähler $\mathcal{A}$-manifolds, Ann. Polon. Math. 75 (2000), 111-124.

[K-S] P. Kobak and A. Swann, The hyperkähler geometry associated to Wolf spaces, Boll. Unione Mat. Ital. Sez. B Artic. Ric. Mat. (8) 4 (2001), 587-595.

[K] M. Konishi, On manifolds with Sasakian 3-structure over quaternion Kähler manifolds, Kōdai Math. Sem. Rep. 26 (1975), 194-200.

[Ku] Y.-Y. Kuo, On almost contact 3-structures, Tôhoku Math. J. 22 (1970), 325-332.

[O'N] B. O'Neill, The fundamental equations of a submersion, Michigan Math. J. 13 (1966), 459-469.

[O-T] J. Oprea and A. Tralle, Symplectic Manifolds with no Kähler Structure, Lecture Notes in Math. 1661, Springer, 1997.

[S] S. Salamon, Quaternionic Kähler manifolds, Invent. Math. 67 (1982), 143-171.

[Sw] A. Swann, Hyperkähler and quaternionic Kähler geometry, Math. Ann. 289 (1991), 421-450. 
[T] S. Tanno, Remarks on a triple of K-contact structures, Tôhoku Math. J. 48 (1996), 519-531.

[W] J. A. Wolf, Complex homogeneous contact manifolds and quaternionic symmetric spaces, J. Math. Mech. 14 (1965), 1033-1047.

Institute of Mathematics

Cracow University of Technology

Warszawska 24

31-155 Kraków, Poland

E-mail: wjelon@usk.pk.edu.pl 